Applied Physiology, Nutrition, and Metabolism

Canadian Science Publishing Physiologie appliquée, nutrition et métabolisme

\title{
Cutaneous vasomotor responses in boys and men
}

\begin{tabular}{|r|l|}
\hline Journal: & Applied Physiology, Nutrition, and Metabolism \\
\hline Manuscript ID & apnm-2018-0083.R1 \\
\hline Danuscript Type: & Article \\
\hline Complete List of Authors: & $\begin{array}{l}\text { Hodges, Gary; Brock University } \\
\text { Mueller, Matthew; Brock University } \\
\text { Cheung, Stephen; Department of Kinesiology, Brock University } \\
\text { Falk, Bareket; Brock University, }\end{array}$ \\
\hline Keyword: & Children, laser-Doppler, local heating, exercise, reactive hyperaemia \\
\hline $\begin{array}{r}\text { Is the invited manuscript for } \\
\text { consideration in a Special } \\
\text { Issue? : }\end{array}$ & N/A \\
\hline
\end{tabular}

SCHOLARONE ${ }^{\text {TH }}$

Manuscripts 


\section{Cutaneous vasomotor responses in boys and men}

2 Gary J. Hodges, Matthew C. Mueller, Stephen S. Cheung, Bareket Falk

3

4 Department of Kinesiology, Brock University, St. Catharines ON L2S 3A1, Canada 5

6 Running head: Cutaneous vasomotor responses in boys and men

\section{Corresponding Author:}

10 Bareket Falk

11 Department of Kinesiology

12 Brock University

13 St. Catharines ON L2S 3A1

14 E-mail: bfalk@brocku.ca

15 Telephone: $1-905-688-5550 \times 4979$ 


\section{Abstract}

17 Few studies have investigated skin blood flow (SkBF) in children and age-related

18 differences in the underlying mechanisms. We examined mechanisms of SkBF responses to

19 local heating, post-occlusive reactive hyperaemia (PORH), and isometric hand-grip exercise

20 (IHE) in adult and prepubescent males, hypothesizing that SkBF responses would be

21 greater in children compared to adults. We measured SkBF in 12 boys ( $9 \pm 1$ y) and 12 men

22 (21 \pm 1 y) using laser-Doppler flowmetry at rest, in response to 3-min PORH, 2-min IHE, and

23 local skin heating to $39^{\circ} \mathrm{C}$ (sub-maximal) and $44^{\circ} \mathrm{C}$ (maximal). Using wavelet analysis we

24 assessed endothelial, neural, and myogenic activities. At rest and in response to local

25 heating to $39^{\circ} \mathrm{C}$, children had higher SkBF and endothelial activity compared to men $(d \geq 1.1$,

$26 p<0.001)$ and similar neurogenic and myogenic activities $(d<0.2, p>0.05)$. Maximal

27 responses to $44^{\circ} \mathrm{C}$ local skin heating, PORH, and IHE did not differ between boys and men

28 (all $d \leq 0.2, p>0.05$ ). During PORH children demonstrated greater endothelial activity

29 compared to men $(d \geq 0.6, p<0.05)$; in contrast, men had higher neurogenic activity $(d=1.0$,

$30 p<0.01$ ). During IHE there were no differences in endothelial, neurogenic, and myogenic

31 activities $(d<0.2, p>0.3)$, with boys and men demonstrating similar increases in endothelial

32 activity and decreases in myogenic activity $(d \geq 0.8, p<0.05)$. These data suggest that boys

33 experience greater levels of SkBF at rest and in response to sub-maximal local heating

34 compared to men, while maximal responses appear to be similar. Additionally, endothelial

35 mediators seem to contribute more to vasodilatation in boys than in men.

37 Keywords: children, laser-Doppler, local heating, exercise, reactive hyperaemia. 


\section{Introduction}

Skin blood flow plays a major role in human thermoregulation, increasing and decreasing flow in response to changes in both core and skin temperature (Johnson et al. 2014). During systemic heat stress, it has been argued that children thermoregulate as effectively as adults. However, while adults rely more on evaporative heat loss, children rely more on enhanced skin blood flow for heat dissipation (Falk and Dotan 2008, Rowland 2008). Reflex thermoregulatory changes in skin blood flow are controlled by two arms of the sympathetic nervous system: an active vasodilator system and a noradrenergic vasoconstrictor system. In adults, the cutaneous vasodilator response to local skin heating 47 (with no change in core temperature) is primarily endothelial-dependent, with endothelial nitric oxide synthase (eNOS) (Kellogg et al. 2008, 2009) and endothelial-derived hyperpolarizing factor (EDHF) (Brunt and Minson 2012) mediating the response. Skin heating to $39^{\circ} \mathrm{C}$ has been shown to be primarily eNOS-dependent (Choi et al. 2014), while

51 local heating to $42^{\circ} \mathrm{C}$ involves a combination of eNOS, EDHF, and neural processes (Johnson

52 et al. 2014). The vasodilator response to local skin heating in children and adolescents has

53 been shown to be larger than in adults (Martin et al. 1995, Hodges et al. 2010). These

54 findings are also supported by the consistent findings that during heat stress, sweating rate 55 is lower in children than adults, suggesting a greater reliance on increasing blood flow to 56 the skin to achieve thermoregulatory balance (Falk and Dotan 2008, Rowland 2008). There 57 have been no studies that have examined the underlying mechanisms of local heating58 induced vasodilatation in healthy children and how they compare to adults.

In adults, isometric exercise elicits physiological responses such as increases in 60 heart rate (HR), mean arterial pressure (MAP), cardiac output $(\dot{Q})$, and sympathetic nerve 61 activity directed to skeletal muscle (Johnson 2010). It has also been reported that isometric 62 exercise leads to an increase in skin sympathetic nerve activity (Johnson 2010). However, 63 in normothermia, the data for forearm skin blood flow responses to isometric exercise 64 appear to be equivocal with both vasoconstriction and vasodilatation being reported (Saad 65 et al. 2001, McCord and Minson 2005, Johnson 2010). There is no work examining the skin 66 blood flow responses to isometric exercise in children and no understanding of the 67 underlying mechanisms eliciting the cutaneous vascular responses. 
The marked, transient increase in microvascular blood flow following arterial occlusion, known as post-occlusive reactive hyperaemia (PORH), is a common assessment of microvascular function and reactivity. The responsible mechanisms governing the cutaneous PORH are unclear, partly because the mediators of this response are not fully understood. Four factors are currently thought to contribute to the PORH response: endothelial vasodilators, metabolic vasodilators, the myogenic response, and sensory

74 nerves. The involvement of endothelial vasodilators is equivocal. That is, nitric oxide and 75 prostaglandins may (Dakak et al. 1998, Binggeli et al. 2003) or may not (Wong et al. 2003, 76 Zhao et al. 2004, Lorenzo and Minson 2007) be mediators of PORH, while EDHFs probably are involved (Lorenzo et al. 2007). There are no studies that have investigated the mechanisms of PORH in children.

The use of spectral analysis of the laser-Doppler signal has enabled non-invasive examination of the mechanisms underlying cutaneous vascular responses (Stefanovska et

81 al. 1999, Kvandal et al. 2006). Previously, invasive and/or pharmacological approaches

82 (intradermal microdialysis and iontophoresis) were necessary to investigate the

83 endothelial and neural components regulating skin blood flow. Use of spectral analysis of

84 the rhythmic variations in cutaneous blood flow enables the examination of specific

85 oscillatory frequencies within the signal to delineate the underlying mechanisms involved

86 in vascular control from the cardiac rhythms $(1 \mathrm{~Hz})$ down to the innate endothelium-

87 related oscillations $(0.01 \mathrm{~Hz})($ Stefanovska et al. 1999). Beyond the ability to compare and contrast the blood flow response, this approach also enables the examination of the mechanisms of action mediating changes in blood flow in children and adults.

There is a dearth of work examining the effects of age and maturation on cutaneous

91 vasodilation in healthy children. Indeed, cutaneous vascular responses to local skin heating,

92 isometric handgrip exercise, and PORH have not been investigated in children relative to

93 adults and neither have the underlying mechanisms. Therefore, the aim of the present

94 study was to examine cutaneous vasomotor responses to PORH, isometric exercise, and

95 local skin heating in the skin of the forearm (non-glabrous) in response to a 3 min reactive

96 hyperaemia, local forearm muscle contraction, and local skin heating respectively, in male

97 youths and young adults. These tests were chosen to potentially provide us with insight

98 regarding mechanistic differences between children and adults: Local heating to $39^{\circ} \mathrm{C}$ is 
99 primarily been shown to be NO-mediated (Choi et al. 2014). PORH is generally considered 100 to be mediated by prostaglandins and EDHF (Wong et al. 2003, Lorenzo and Minson 2007).

101 Isometric response is thought to be neurally-mediated (Crandall et al. 1995, Saad et al.

102 2001). Thus, these 3 assessments were intended to provide us with some insight into NO-

103 dependent, NO-independent, and neural mechanisms and how they might differ between

104 children and adults. We hypothesize that SkBF responses at rest and in response to local

105 heating, PORH, IHE will be greater than those observed in adults. We hypothesize that

106 these larger responses in boys compared to men will be mediated by greater endothelial

107 and neurogenic contributions.

108

109 


\section{Materials and methods}

\section{Participants}

112 Twelve healthy men and 12 healthy boys participated in the study. They did not

113 have any overt symptoms or history of cardiovascular disease, and were not taking any

114 medications. The study was cleared by Brock University's Bioscience Ethics Review Board

115 (\#16-026) and adhered to the Declaration of Helsinki. Prior to participating in this study,

116 adult participants gave written and verbal consent, whereas both children participants and

117 their parents gave written and verbal consent. Participant characteristics are presented in

118 Table 1. No differences were observed between groups in relative maximal oxygen uptake

$119\left(\dot{V} \mathrm{O}_{2 \max }\right)$ and both groups were of normal-weight.

121 Procedure

122 Each participant visited the laboratory twice. The first visit involved familiarization

123 with study equipment and procedures, anthropometric measurements (mass and stature)

124 and an incremental cycling test to volitional exhaustion to determine $\dot{V} \mathrm{O}_{2 \text { max. }}$ The second

125 visit was the experimental session in which the cutaneous blood flow response was

126 investigated in response to isometric exercise, PORH and local heating, as outlined in

127 Figure 1.

Measurements

130 Laser-Doppler flowmetry was used for an index of forearm skin blood flow

131 (PeriFlux 5010 laser-Doppler perfusion monitor; Perimed; Järfälla, Sweden), and was

132 expressed as laser-Doppler flux (LDF). LDF provides an accurate index of skin blood flow

133 and is not influenced by underlying skeletal muscle blood flow (Saumet et al. 1988). An

134 integrated laser-Doppler local heating probe (Probe 413; Perimed) was used. A calibration

135 device (PF 1000, Perimed) standard was used to adjust the laser-Doppler fluxmeter

136 readings to coincide with the readings obtained with Perimed's Motility Standard.

137 Manipulation of local skin temperature $\left(T_{\text {loc }}\right)$ was achieved with local heating unit and

138 heating probe holders (PF5020 local heating units and PeriFlux 5020 Temperature Unit;

139 Perimed) that controlled and monitored skin surface temperature. 
Isometric exercise

Prior to data collection, three maximal voluntary contractions (MVC) were

142 performed. The maximal response was used to determine the MVC. The participant

143 performed a 2-min isometric handgrip exercise at 30\% MVC with the LDF probe

144 maintaining $T_{\text {loc }}$ at $33^{\circ} \mathrm{C}$.

145

Post-occlusive reactive hyperaemia

Post-occlusive reactive hyperaemia (PORH) was performed, as previously described 148 (Tew et al. 2012, Hodges et al. 2017c), by inflating a size-appropriate blood pressure cuff 149 on the upper arm (bicep) to $230 \mathrm{~mm} \mathrm{Hg}$ and maintaining that pressure for $3 \mathrm{~min}$. The cuff 150 was then rapidly deflated and a 5-min recovery period was recorded. Ten to fifteen

151 minutes of additional time ensured resting levels of skin blood flow were achieved.

153 Local skin heating

154 Local skin heating was performed in two stages. First, following baseline

155 measurements at a thermoneutral temperature of $33^{\circ} \mathrm{C}$. Second, $T_{\text {loc }}$ was increased to $39^{\circ} \mathrm{C}$

156 at a rate of $3^{\circ} \mathrm{C} \cdot \mathrm{min}^{-1}$ and held for a minimum $25 \mathrm{~min}$ or until a stable plateau had been

157 established. In adults, the cutaneous vasodilatation to this level of local skin heating has

158 been reported to be primarily eNOS-mediated (Choi et al. 2014). Finally, $T_{\text {loc }}$ was increased

159 from 39 to $44^{\circ} \mathrm{C}$ at a rate of $1^{\circ} \mathrm{C} \cdot \mathrm{min}^{-1}$ and held for a minimum of $25 \mathrm{~min}$. This level of local

160 heating elicits a maximal thermal vasodilatation in adults that is dependent on many

161 factors including endothelial, neural, and other sources (Wong and Fieger 2010, Johnson et

162 al. 2014, Hodges and Sparks 2014, Hodges and Del Pozzi 2014). Each duration of

163 measurement outlined above and in Figure 1 was a minimum, with each level of heating

164 maintained until a stable plateau in LDF was achieved.

166 Determination of $\dot{V} \mathrm{O}_{2} \max$.

167 Participants began with a 3-5-minute warm-up and cadence familiarization, 168 followed by the graded exercise to exhaustion. Boys typically started at 25-35 W and 169 increased by $10 \mathrm{~W} \cdot \mathrm{min}^{-1}$, while men typically began the graded protocol at $60-70 \mathrm{~W}$ and 
170 increased by $10 \mathrm{~W} \cdot \mathrm{min}^{-1}$. The test continued to volitional exhaustion. As has previously

171 demonstrated by Barker et al. (2011) we did not rely on the commonly-used fixed criteria

172 for $\dot{V} \mathrm{O}_{2}$ max attainment (e.g., $90 \%$ predicted max heart rate (HR), or respiratory exchange

173 ratio of 1.05 ), but rather exceeded them in motivating the participants and verbally

174 encouraging them to reach their respective utmost exhaustion. To verify that the testing

175 protocol indeed elicited highest possible values, supra-maximal testing at $105 \%$ of the peak

$176 \dot{V} \mathrm{O}_{2}$ test's final power, was administered, $\sim 10 \mathrm{~min}$ after the graded exercise protocol (as

177 suggested by Barker et al. (2011)). In no case was an improvement observed relative to the

178 preceding $\dot{V} \mathrm{O}_{2}$ max test. $\dot{V} \mathrm{O}_{2}$ max was recorded as the average of the highest three

179 consecutive 15-s intervals near the end of the volitional exercise test. Expired gas was

180 collected and analyzed using the Moxus metabolic cart (AEI Technologies, PA, USA),

181 calibrated prior to each test. A cadence of $80 \mathrm{rpm}$ or higher was required throughout each

182 test. The metabolic cart could be switched between standard (adult) and small (pediatric)

183 mixing chambers. The latter was used for children of less than $\sim 40 \mathrm{~kg}$ body mass.

Other cardiovascular measurements

HR was calculated from R-R intervals that were collected using a standard threelead electrocardiogram (BioAmp, ADInstruments, Colorado Springs, USA). Blood pressure, was recorded by auscultation from the contralateral arm every $30 \mathrm{~min}$ by the same investigator. Additionally, photoplethysmography (Nexfin, Edwards Lifesciences, Irvine, USA) was used to continuously calculate blood pressure and cardiac output $(\dot{Q})$ from finger

191 perfusion. Mean arterial pressure (MAP) was calculated as 0.33 systolic pressure +0.67

192 diastolic pressure.

Wavelet transform

A Morlet mother wavelet was used to perform wavelet analysis on the LDF signal.

196 Wavelet analysis was chosen as it provides good time and frequency resolution within the

197 uncertainty principle. It uses an adjustable window to provide good frequency resolution

198 for lower and higher frequencies by using a longer and shorter analysis window,

199 respectively. This method characterizes the dynamics of signals over a wide frequency 
200 range, from 0.005 to $1.6 \mathrm{~Hz}$. The six frequency intervals and associated physiological

201 functions were as follows: $0.005-0.01 \mathrm{~Hz}$ (endothelial: NO-independent) and 0.01-0.02 Hz

202 (endothelial: NO-dependent) (Kvandal et al. 2006); 0.02-0.05 Hz (neurogenic) (Söderström

203 et al. 2003, Hodges et al. 2017a); 0.05-0.15 Hz (myogenic) (Johnson 1989, Söderström et al.

204 2003); 0.15-0.40 Hz (respiratory); 0.40-1.6 Hz (cardiac).

Data collection and statistical analysis

Laser-Doppler flowmetry (LDF) and finger photoplethysmography data were collected at $40 \mathrm{~Hz}$, local skin temperature data were collected at $4 \mathrm{~Hz}$, and ECG data were collected at $1 \mathrm{KHz}$ (LabChart, ADInstruments). The data from the entire protocol $\sim 95 \mathrm{~min}$ was exported and run through the wavelet transform in a custom written computer script

211 (Iatsenko et al. 2015) (Matlab®, The MathWorks Inc., Natick, MA, USA). Subsequently, 212 median amplitudes were chosen from the appropriate section (Fig. 1) such that data for 213 basal (4 min), hand grip (final $20 \mathrm{~s}$ ), recovery from hand grip (final $20 \mathrm{~s}$ ), PORH (10 s), local

214 heating at $39^{\circ} \mathrm{C}$ and $44^{\circ} \mathrm{C}(4 \mathrm{~min})$ were extracted. The same sections were extracted as 215 means for laser-Doppler data.

Analysis of time segments of $20 \mathrm{~min}$ is needed for good low-frequency resolution

217 and detection of oscillations in the low-frequency range (Stefanovska et al. 1999). We ran

218 the wavelet transform on a minimum of $80 \mathrm{~min}$ of data. While we have chosen short time

219 windows for certain phases to extract median amplitudes of the vasodilator responses

220 (handgrip, PORH), the fact that the wavelet was run on the entire data laser-Doppler signal

221 ensured that even these smaller extracted portions had appropriate resolution and power

222 to examine the low-frequency bands (Hodges et al. 2017b, 2017a).

223 Laser-Doppler flowmetry data were normally distributed as assessed by the

224 Shapiro-Wilk test of normality. Data are presented as cutaneous vascular conductance (CVC

$\left.225=\mathrm{LDF} \cdot \mathrm{MAP}^{-1}\right)$. $\mathrm{CVC}$ data are presented as raw $\left(\mathrm{PU} \cdot \mathrm{mm} \mathrm{Hg}^{-1}\right.$ ) and normalized to maximal

226 thermal vasodilation, local skin heating to $44^{\circ} \mathrm{C}$ (\%max). Isometric handgrip CVC data were

227 analyzed by repeated measures ANOVA with a Dunnett's multiple-comparison test. Two-

228 way repeated measures ANOVA (group $\mathrm{x}$ temperature) compared CVC responses in boys

229 and men at $33^{\circ} \mathrm{C}$ and $39^{\circ} \mathrm{C}$, with a Bonferroni post-hoc correction for multiple comparisons. 
230 Vasomotion data (AU) were normally distributed as assessed by the same criteria above. A 231 two-way repeated measures ANOVA (group $\mathrm{x}$ temperature) was used to examine local 232 heating responses (baseline, $39^{\circ} \mathrm{C}$, and $44^{\circ} \mathrm{C}$ ) with a Bonferroni post-hoc correction for 233 multiple comparisons. Participant characteristics are provided as mean and SD, while all 234 other data are presented as mean and 95\%CI. Analyses were performed using Graphpad 235 Prism (v6.01, GraphPad Software Inc., La Jolla, CA, USA).

236 Cohen's $d$ (Cohen 1988) was used to calculate effect sizes using ESCI-delta (Geoff 237 Cumming, La Trobe University, Australia), and descriptors for magnitudes used is based 238 upon those of Sawilowsky (Sawilowsky 2009): Very small 0.01, Small 0.2, Medium 0.5, 239 Large 0.8, Very large 1.20, Huge 2.00. 


\section{$240 \quad$ Results}

241 Basal and local heating responses

242 There were main effects for group and heating level (both $p<0.05$ ). Basal CVC was

243 higher in the boys $\left(0.225 \pm 0.028 \mathrm{PU} \cdot \mathrm{mmHg}^{-1}\right)$ than in the men $\left(0.102 \pm 0.0237 \mathrm{PU} \cdot \mathrm{mmHg}^{-1}\right)$

$244(d=2.7, p<0.001)$ (Fig. 2A). This was also the case for CVC normalized to the maximal

245 response $(12 \pm 2 \% \max v s .6 \pm 2 \% \max )(d=1.4, p=0.002)$ (Fig. 2B). We also observed greater

$246 \mathrm{CVC}$ response to local heating of $39^{\circ} \mathrm{C}$ in the boys compared to the men in both raw $\mathrm{CVC}$

$247\left(0.442 \pm 0.099\right.$ vs. $\left.0.268 \pm 0.073 \mathrm{PU} \cdot \mathrm{mmHg}^{-1}\right)(d=1.1, p=0.01)$ and normalized to $\max (23 \pm 4$

$248 \% \max v s .15 \pm 4 \% \max )(d=1.0, p=0.019)$. Boys had higher basal responses (Table 2). When

249 the responses to $39^{\circ} \mathrm{C}$ were expressed as percent change from baseline, there was no

250 difference between the boys and men $(125 \pm 81 \% v s .183 \pm 80 \% ; d=0.3, p=0.13)$. There were

251 no differences between the groups in raw CVC during local heating at $44^{\circ} \mathrm{C}(d=0.2, p=0.6)$.

252 Laser-Doppler flux and wavelet analysis of the local heating responses are

253 presented in Table 2. Laser-Doppler flux comparisons between boys and men mirror those

254 of CVC in that we observed main effects for group and heating level $(p<0.05)$, but no

255 interaction ( $p>0.05)$. For the vasomotion analyses, there were main effects for group,

256 heating level, and interaction $(p<0.05)$. Under basal conditions, men have stronger

257 neurogenic and myogenic components than boys (all $d \geq 0.9, p<0.05$ ); whereas, endothelial

258 NO-independent and NO-dependent influences appear to have a greater role in boys (both $259 d \geq 0.8, p<0.05)$. In response to local heating to $39^{\circ} \mathrm{C}$ both groups present with increased NO-

260 independent endothelial activity (both $d \geq 1.1, p<0.05$ ). In response to skin heating to $44^{\circ} \mathrm{C}$

261 we observed that cardiac influence increased (both $d \geq 1.2, p<0.05$ ) in both groups and

262 became the main contributing component to the vasomotor response.

264 Post-occlusive reactive hyperaemia

265 There was no difference in the maximal CVC responses to PORH between the boys

266 and men, whether expressed in absolute units or as a percentage of the maximal CVC (both $267 d \leq 0.1, p>0.3)$ (Table 3). However, both time-to-peak $(d=2.9, p<0.001)$ and area-under-the-

268 curve (AUC) $(d=2.2, p<0.001)$ of the PORH were greater in boys compared to men. 
There was no difference in the maximal LDF response between the boys and men $(d=0.3, p=0.2$ ) (Table 4). However, both endothelial NO-independent and NO-dependent

271 activities were higher in the children (both $d \geq 0.6, p<0.05$ ); while neurogenic, respiratory,

272 and cardiac frequency bands were higher in the men compared to the boys (all $d \geq 0.8$,

$273 p<0.05)$.

274

275 Isometric handgrip exercise

276 During the isometric handgrip exercise trials, cutaneous measurements (LDF, raw

277 CVC, and CVC \%max) were significantly greater in the boys compared with men (Fig. 3). In

278 absolute values, MAP and $\dot{Q}$ were lower in boys compared to men (all $d>0.8, p<0.05$ ), but

279 when expressed as changes from baseline, there were no differences between boys and 280 men (all $d<0.2, p>0.05)$; HR responses did not differ $(d<0.2, p>0.05)$. Endothelial and 281 neurogenic responses to exercise were greater in the men compared to the boys (both $282 d \geq 0.8, p<0.05$ ) (Table 5).

283 Isometric handgrip exercise caused a significant increase in both boys and men in 284 LDF, MAP, and HR (all $d>0.8, p<0.05$ ). When cutaneous vasomotor responses were 285 expressed as CVC, during isometric exercise, there was a marked vasoconstriction only in 286 the boys (all $d>0.8, p<0.05$ ) while there was no change in the men (Fig. 3). $\dot{Q}$ was

287 unchanged during the exercise in both men and boys $(d<0.2, p>0.05)$. Isometric exercise 288 caused similar increases in NO-independent endothelial activity and decreases in myogenic 289 activity in both men and boys groups (all $d>0.8, p<0.05$ ) (Table 5).

290 Post-handgrip, there were abrupt, transient increases in CVC in boys $(d>0.8$, $291 p<0.05)$. In the men, $\dot{Q}$ was also temporarily increased relative to the exercise and pre-

292 exercise baseline $(d=0.7, p=0.006)$. LDF, MAP, and HR all decreased post-exercise in both 293 men and boys (all $d>0.8, p<0.05$ ). 


\section{Discussion}

We used a combination of laser-Doppler flowmetry and wavelet analysis of the LDF signal to non-invasively examine mechanisms of cutaneous vascular function in both adult and prepubescent males. Cutaneous vascular responses and endothelial activity at rest and in response to local skin heating to $39^{\circ} \mathrm{C}$ as well as during PORH were greater in boys compared to men; while men had larger neurogenic responses. Maximal LDF responses to

$30244^{\circ} \mathrm{C}$ local skin heating, PORH, and isometric handgrip exercise did not differ between boys 303 and men and both boys and men presented with similar increases in endothelial,

304 neurogenic, and myogenic activity. These data suggest that, despite similar maximal

305 responses, children respond with a greater level of vasodilatation at rest and to

306 submaximal stimuli compared to men. Additionally, endothelial-mediators seem to be the

307 predominant mediators of vasodilatation in boys. Finally, we demonstrate that the

308 combination of laser-Doppler flowmetry and wavelet analysis to non-invasively examine 309 mechanisms of cutaneous vascular function can be successfully applied in children.

\section{Local heating}

312 Forearm skin vascular conductance, expressed both as CVC and CVC normalized to 313 maximum $\left(44^{\circ} \mathrm{C}\right)$, was higher in boys compared with men at both $33^{\circ} \mathrm{C}$ and NO-mediated

$31439^{\circ} \mathrm{C}$ local heating (Choi et al. 2014), suggesting enhanced microvascular endothelial

315 dilation. This is consistent with previous reports of higher skin blood flow at rest and 316 during local heating (Martin et al. 1995, Hodges et al. 2010) in children and adolescents

317 compared with adults. It is also consistent with the observed greater increase in skin blood 318 flow in younger compared with older boys during systemic heat stress [exercise in the heat

319 (Falk et al. 1992)], and the assumption of greater reliance on dry heat exchange in children 320 compared with adults (Falk and Dotan 2008, Rowland 2008). Note that there were no

321 difference in fitness level $\left(\dot{V} \mathrm{O}_{2 \max }\right)$ between the groups and no participants were

322 overweight or obese, both of which can affect endothelial function in both children and 323 adults (Abbott et al. 2002, Roche et al. 2010).

324 At rest under thermoneutral conditions $\left(33^{\circ} \mathrm{C}\right)$ and in response to submaximal local

325 heating, boys had greater NO-dependent and NO-independent activities than the men. 
326 Consistent with previous reports in adults, endothelial activity plays an important role

327 under basal thermoneutral conditions (Del Pozzi et al. 2013, Hodges and Sparks 2014) and

328 in response to local skin heating to $39^{\circ} \mathrm{C}$ (Choi et al. 2014). In general, the endothelial

329 response, both NO-dependent and -independent, was greater in children, and it would

330 seem that boys are more responsive or are more sensitive to increases in skin temperature

331 than men. This greater sensitivity may be due to higher responsiveness of the mechanisms

332 governing vasodilatation, specifically of the endothelial tissue. Indeed, this postulate is

333 consistent with the greater endothelial-mediated cutaneous vasodilator response to local

334 skin heating in young vs. older men (Hodges et al. 2017b) and may imply that the 'aging

335 process' of the endothelium begins at a very young age. This is consistent with the age-

336 related decreases in skin blood flow in response local heating seen in large cross-sectional

337 studies (Martin et al. 1995, Hodges et al. 2010) and with the maturity-related lower skin

338 blood flow observed in late- compared with pre-pubertal boys (Falk et al. 1992). Also, it is

339 important to note that the vasodilator responses to $39^{\circ} \mathrm{C}$ heating observed in this study

340 were about half the size of those reported in the Choi et al (Choi et al. 2014) study. Whether

341 this is contributing to the reduced endothelial-signal in men compared to boys is unclear

342 and warrants further investigation.

343 It is of note that the cardiac interval $(0.4-1.6 \mathrm{~Hz})$ was largest during local heating at

$34444^{\circ} \mathrm{C}$. We do not consider this observation to mean that cardiac factors were the mediating

345 force behind this vasodilatory response. Rather, the large vasodilatory response will mean

346 the vascular smooth muscle will be almost maximally-relaxed (flux has increased $>10$ times

347 compared to basal measures), and the larger oscillations in blood flow that occur between

348 systole to diastole are what are contributing more to the overall power of the signal in the

349 cardiac interval.

350

351 Post-Occlusive Reactive Hyperaemia (PORH)

352 There was no difference in the maximal PORH response between boys and men;

353 however, AUC was greater in boys, as was the time-to-peak. As was the case with local

354 heating, endothelial (both NO-dependent and independent) activity was higher in boys.

355 This is an interesting observation as previous work in adults has shown that the 
356 mechanisms responsible for PORH are unclear, partly because the mediators of this

357 response are not fully understood and the data are inconsistent (Johnson et al. 2014). The

358 role of endothelial-derived vasodilators in adults is equivocal. Nitric oxide and

359 prostaglandins may (Dakak et al. 1998, Binggeli et al. 2003) or may not (Wong et al. 2003,

360 Zhao et al. 2004, Lorenzo and Minson 2007) be mediators of PORH, while EDHFs probably

361 are involved (Lorenzo and Minson 2007). Shear stress stimulation, the primary vasodilator

362 action of reactive hyperaemia, has been shown to increase prostaglandin levels, leading to

363 improved vascular function separate from any contribution of nitric oxide (Koller et al.

364 1994; Walshe et al. 2005). We recently published work that showed that shear stress leads

365 to a larger peak and AUC PORH responses in healthy adults (Hodges et al. 2017c). Thus, the

366 present data indicate that boys have greater endothelial response (both NO and non-NO

367 sources) in the hyperemic response to brief occlusion, which is consistent with the greater

368 endothelial involvement to local skin heating.

369 A curious observation is that the time-to-peak for the PORH response was longer in

370 the boys than in the men. This was an unexpected finding, as usually the kinetics

371 (neuromuscular and metabolic) are faster in children compared to adults (Falk and Dotan

372 2008, Black et al. 2008, Falk et al. 2009, Mitchell et al. 2011). This finding might be a

373 consequence of the greater reliance on endothelial-dependent vasodilators and a smaller

374 contribution of neural input, both sensory and sympathetic (Larkin and Williams 1993,

375 Lorenzo and Minson 2007) in boys compared to men (Table 4), such that these metabolic

376 signals that take longer to elicit vascular responses. Furthermore, the AUC was greater in

377 the boys compared to the men. This might be due to a heavier reliance on the endothelial

378 dilators (NO, $\mathrm{PG}_{\mathrm{i}}$, and $\mathrm{EDHF}$ ) rather than on neural components in boys. Prior work has

379 shown that in adults, endothelial dilators are less important in adults than neural inputs

380 (Wong et al. 2003, Zhao et al. 2004).

382 Isometric exercise

383 In both boys and men, the contribution of both endothelial-NO-dependent and NO-

384 independent activities increased with exercise while other factors decreased or did not

385 change. Thus, it would appear that similar mechanisms were employed by both boys and 
386 men to elicit the vasodilatation that occurred during isometric handgrip exercise. Our study

387 supports previous work which reported mild increase in forearm skin vascular

388 conductance during isometric exercise in adults (Saad et al. 2001, McCord and Minson

3892005 ) and extend these findings to boys. Importantly, this is the first study to demonstrate

390 that in boys the increases in forearm skin vascular conductance during isometric exercise is

391 due to a combination of an increase in both NO-dependent and NO-independent endothelial

392 activity and a reduction in basal sympathetic activity (i.e. sympathetic withdrawal).

Limitations

395 We did not directly inhibit endothelial enzymes, block sensory and sympathetic

396 nerves, or antagonise receptors. However, wavelet analysis is considered an excellent tool

397 to study the mechanisms of vasomotor responses (Stefanovska et al. 1999). It is

398 particularly attractive for the use in children and other vulnerable populations, because it

399 is non-invasive and does not require the use of pharmacological agents. However, its

400 specific accuracy in these populations is not clear. Additionally, wavelet analysis may be

401 unable to delineate local and reflex sympathetic activity. While previous work has clearly

402 demonstrated that the neural frequency band $(0.02-0.05 \mathrm{~Hz})$ is mostly sympathetic

403 (Hodges et al. 2017a), there is also likely to be some sensory nerve involvement. Thus,

404 categorical statement of what the neural influences are and whether the observed effects

405 are of local or reflex origin on cutaneous vascular control are not possible at the present

406 time with this approach. The observation of an increase in myogenic activity with local

407 heating is puzzling. Data supporting the vascular oscillation centred around $0.1 \mathrm{~Hz}$ and

408 termed myogenic activity is still equivocal. A sympathetic origin of these oscillations has

409 been suggested (Stauss et al. 1998, Cevese et al. 2001); however, studies employing

410 anaesthesia and skin flaps (Söderström et al. 2003) and with localized sympathetic

411 blockade (Hodges et al. 2017a) have been able to uncouple the systems and distinguish

412 separate oscillations. However, it is important to note that there has been no direct

413 confirmation of the role of myogenic activity due to the difficulties in manipulating and

414 measuring microvascular pressure and obtaining a flow measure simultaneously in vivo in

415 human skin. That is why we have focused mostly on oscillations associated with 
416 endothelial and neural activities, as these have the most evidence to support their function

417 (Kvandal et al. 2003, 2006, Hodges et al. 2017a). Also, it is important to note that the

418 frequency bins are not completely independent and caution must be exercised in making

419 definitive statements regarding the underlying mechanisms of action. We did not

420 randomize the order of tests. We waited a minimum of $10 \mathrm{~min}$ between each assessment,

421 but there could have been an order effect. and importantly, this could be different between

422 the two groups. Finally, we assessed vasomotor function in males only. While previous

423 work has shown no effect of menstrual status on cutaneous vasomotor responses using

424 FFT analysis (Rossi et al. 2009), future work should examine cutaneous vasomotor function

425 in maturing females, as the influence of female sex-hormones may differ than that of

426 androgens.

427 In summary, we have found that at rest and in response to submaximal local skin

428 heating, boys have higher skin blood flow than men and are more reliant on endothelial-

429 dependent mechanisms. Maximal local heating to $44^{\circ} \mathrm{C}$, reactive hyperemic, and isometric

430 hand-grip responses do not differ between boys and men. However, during these stimuli,

431 endothelial-mediators are the predominant mediators of vasodilatation in boys, while in

432 the men, neural and myogenic responses appear to play a larger role. Also, we have

433 demonstrated that the combination of laser-Doppler and wavelet analysis to non-invasively

434 examine mechanisms of cutaneous vascular function can be successfully applied in

435 children.

\section{Acknowledgements}

438 We thank the participants and parents for volunteering their time and effort for the

439 study. MCM was supported by a Canadian Institute for Health Research (CIHR)

440 undergraduate award. SSC was supported by a Canada Research Chair.

\section{Author contributions}

443 GJH conceived and designed the study. GJH and MCM collected and analysed the 444 data. GJH, MCM, SSC, and BF interpreted the data. GJH drafted the manuscript. MCM, SSC, 445 and BF revised the manuscript critically for important intellectual content. All authors 
446 approved the final version of the manuscript and agree to be accountable for all aspects of 447 the work.

448

\section{Conflict of interest}

450 The authors have no conflicts of interest to report. 


\section{References}

Abbott, R.A., Harkness, M.A., and Davies, P.S.W. 2002. Correlation of habitual physical activity levels with flow-mediated dilation of the brachial artery in 5-10 year old children. Atherosclerosis 160(1): 233-239.

Barker, A.R., Williams, C.A., Jones, A.M., and Armstrong, N. 2011. Establishing maximal oxygen uptake in young people during a ramp cycle test to exhaustion. Br. J. Sports Med. 45(6): 498-503. doi:10.1136/bjsm.2009.063180.

Binggeli, C., Spieker, L.E., Corti, R., Sudano, I., Stojanovic, V., Hayoz, D., Lüscher, T.F., and Noll, G. 2003. Statins enhance postischemic hyperemia in the skin circulation of hypercholesterolemic patients: a monitoring test of endothelial dysfunction for clinical practice? J. Am. Coll. Cardiol. 42(1): 71-77.

Black, M.A., Green, D.J., and Cable, N.T. 2008. Exercise prevents age-related decline in nitric-oxidemediated vasodilator function in cutaneous microvessels. J. Physiol. 586(14): 3511-3524. doi:10.1113/jphysiol.2008.153742.

Brunt, V.E., and Minson, C.T. 2012. KCa channels and epoxyeicosatrienoic acids: major contributors to thermal hyperaemia in human skin. J. Physiol. 590(15): 3523-3534. doi:10.1113/jphysiol.2012.236398.

Cevese, A., Gulli, G., Polati, E., Gottin, L., and Grasso, R. 2001. Baroreflex and oscillation of heart period at $0.1 \mathrm{~Hz}$ studied by alpha-blockade and cross-spectral analysis in healthy humans. J. Physiol. 531(Pt 1): 235-244.

Choi, P.J., Brunt, V.E., Fujii, N., and Minson, C.T. 2014. New approach to measure cutaneous microvascular function: an improved test of NO-mediated vasodilation by thermal hyperemia. J. Appl. Physiol. Bethesda Md 1985 117(3): 277-283. doi:10.1152/japplphysiol.01397.2013.

Cohen, J. 1988. Statistical power analysis for the behavioral sciences. Lawrence Erlbaum, Hillsdale, NJ, USA.

Crandall, C.G., Musick, J., Hatch, J.P., Kellogg, D.L., and Johnson, J.M. 1995. Cutaneous vascular and sudomotor responses to isometric exercise in humans. J. Appl. Physiol. Bethesda Md 1985 79(6): 1946-1950. doi:10.1152/jappl.1995.79.6.1946.

Dakak, N., Husain, S., Mulcahy, D., Andrews, N.P., Panza, J.A., Waclawiw, M., Schenke, W., and Quyyumi, A.A. 1998. Contribution of nitric oxide to reactive hyperemia: impact of endothelial dysfunction. Hypertens. Dallas Tex 1979 32(1): 9-15.

Del Pozzi, A.T., Carter, S.J., Collins, A.B., and Hodges, G.J. 2013. The regional differences in the contribution of nitric oxide synthase to skin blood flow at forearm and lower leg sites in response to local skin warming. Microvasc. Res. 90: 106-111.

Falk, B., Bar-Or, O., and MacDougall, J.D. 1992. Thermoregulatory responses of pre-, mid-, and latepubertal boys to exercise in dry heat. Med. Sci. Sports Exerc. 24(6): 688-694.

Falk, B., Brunton, L., Dotan, R., Usselman, C., Klentrou, P., and Gabriel, D. 2009. Muscle strength and contractile kinetics of isometric elbow flexion in girls and women. Pediatr. Exerc. Sci. 21(3): 354364.

Falk, B., and Dotan, R. 2008. Children's thermoregulation during exercise in the heat: a revisit. Appl. Physiol. Nutr. Metab. Physiol. Appl. Nutr. Metab. 33(2): 420-427. doi:10.1139/H07-185.

Hodges, G.J., and Del Pozzi, A.T. 2014. Noninvasive examination of endothelial, sympathetic, and myogenic contributions to regional differences in the human cutaneous microcirculation. Microvasc. Res. 93: 87-91. doi:10.1016/j.mvr.2014.04.002.

Hodges, G.J., Mallette, M.M., Martin, Z.T., and Del Pozzi, A.T. 2017a. Effect of sympathetic nerve blockade on low-frequency oscillations of forearm and leg skin blood flow in healthy humans. Microcirc. N. Y. N 1994 24(7). doi:10.1111/micc.12388. 
Hodges, G.J., Mallette, M.M., Tew, G.A., Saxton, J.M., Moss, J., Ruddock, A.D., and Klonizakis, M. 2017b. Effect of age on cutaneous vasomotor responses during local skin heating. Microvasc. Res. 112: 47-52. doi:10.1016/j.mvr.2017.03.002.

Hodges, G.J., Sharp, L., Clements, R.E., Goldspink, D.F., George, K.P., and Cable, N.T. 2010. Influence of age, sex, and aerobic capacity on forearm and skin blood flow and vascular conductance. Eur. J. Appl. Physiol. 109(6): 1009-1015. doi:10.1007/s00421-010-1441-7.

Hodges, G.J., and Sparks, P.A. 2014. Noradrenaline and neuropeptide Y contribute to initial, but not sustained, vasodilatation in response to local skin warming in humans. Exp. Physiol. 99(2): 381392. doi:10.1113/expphysiol.2013.075549.

Hodges, G.J., Stewart, D.G., Davison, P.J., and Cheung, S.S. 2017c. The role of shear stress on cutaneous microvascular endothelial function in humans. Eur. J. Appl. Physiol. 117(12): 2457-2468. doi:10.1007/s00421-017-3732-8.

Iatsenko, D., McClintock, P.V.E., and Stefanovska, A. 2015. Linear and synchrosqueezed time-frequency representations revisited: Overview, standards of use, resolution, reconstruction, concentration, and algorithms. Digit. Signal Process. 42: 1-26. doi:10.1016/j.dsp.2015.03.004.

Johnson, J.M. 2010. Exercise in a hot environment: the skin circulation. Scand. J. Med. Sci. Sports 20 Suppl 3: 29-39. doi:10.1111/j.1600-0838.2010.01206.x.

Johnson, J.M., Minson, C.T., and Kellogg, D.L. 2014. Cutaneous vasodilator and vasoconstrictor mechanisms in temperature regulation. Compr. Physiol. 4(1): 33-89. doi:10.1002/cphy.c130015.

Johnson, P.C. 1989. The myogenic response in the microcirculation and its interaction with other control systems. J. Hypertens. Suppl. Off. J. Int. Soc. Hypertens. 7(4): S33-39; discussion S40.

Kellogg, D.L., Zhao, J.L., and Wu, Y. 2008. Endothelial nitric oxide synthase control mechanisms in the cutaneous vasculature of humans in vivo. Am. J. Physiol. Heart Circ. Physiol. 295(1): H123-129. doi:10.1152/ajpheart.00082.2008.

Kellogg, D.L., Zhao, J.L., and Wu, Y. 2009. Roles of nitric oxide synthase isoforms in cutaneous vasodilation induced by local warming of the skin and whole body heat stress in humans. J. Appl. Physiol. Bethesda Md 1985 107(5): 1438-1444. doi:10.1152/japplphysiol.00690.2009.

Kvandal, P., Landsverk, S.A., Bernjak, A., Stefanovska, A., Kvernmo, H.D., and Kirkebøen, K.A. 2006. Lowfrequency oscillations of the laser Doppler perfusion signal in human skin. Microvasc. Res. 72(3): 120-127. doi:10.1016/j.mvr.2006.05.006.

Kvandal, P., Stefanovska, A., Veber, M., Kvernmo, H.D., Kvermmo, H.D., and Kirkebøen, K.A. 2003. Regulation of human cutaneous circulation evaluated by laser Doppler flowmetry, iontophoresis, and spectral analysis: importance of nitric oxide and prostaglandines. Microvasc. Res. 65(3): 160171.

Larkin, S.W., and Williams, T.J. 1993. Evidence for sensory nerve involvement in cutaneous reactive hyperemia in humans. Circ. Res. 73(1): 147-154.

Lorenzo, S., and Minson, C.T. 2007. Human cutaneous reactive hyperaemia: role of BKCa channels and sensory nerves. J. Physiol. 585(Pt 1): 295-303. doi:10.1113/jphysiol.2007.143867.

Martin, H.L., Loomis, J.L., and Kenney, W.L. 1995. Maximal skin vascular conductance in subjects aged 585 yr. J. Appl. Physiol. Bethesda Md 1985 79(1): 297-301. doi:10.1152/jappl.1995.79.1.297.

McCord, G.R., and Minson, C.T. 2005. Cutaneous vascular responses to isometric handgrip exercise during local heating and hyperthermia. J. Appl. Physiol. Bethesda Md 1985 98(6): 2011-2018. doi:10.1152/japplphysiol.00888.2004.

Mitchell, C., Cohen, R., Dotan, R., Gabriel, D., Klentrou, P., and Falk, B. 2011. Rate of muscle activation in power- and endurance-trained boys. Int. J. Sports Physiol. Perform. 6(1): 94-105.

Roche, D.M., Rowland, T.W., Garrard, M., Marwood, S., and Unnithan, V.B. 2010. Skin microvascular reactivity in trained adolescents. Eur. J. Appl. Physiol. 108(6): 1201-1208. doi:10.1007/s00421009-1328-7. 
Rossi, M., Di Maria, C., Erba, P., Galetta, F., Carpi, A., and Santoro, G. 2009. Study of skin vasomotion during phollicular and luteal phase in young healthy women. Clin. Hemorheol. Microcirc. 42(2): 107-115. doi:10.3233/CH-2009-1189.

Rowland, T. 2008. Thermoregulation during exercise in the heat in children: old concepts revisited. J. Appl. Physiol. Bethesda Md 1985 105(2): 718-724. doi:10.1152/japplphysiol.01196.2007.

Saad, A.R., Stephens, D.P., Bennett, L.A., Charkoudian, N., Kosiba, W.A., and Johnson, J.M. 2001. Influence of isometric exercise on blood flow and sweating in glabrous and nonglabrous human skin. J. Appl. Physiol. Bethesda Md 1985 91(6): 2487-2492. doi:10.1152/jappl.2001.91.6.2487.

Saumet, J.L., Kellogg, D.L., Taylor, W.F., and Johnson, J.M. 1988. Cutaneous laser-Doppler flowmetry: influence of underlying muscle blood flow. J. Appl. Physiol. Bethesda Md 1985 65(1): 478-481. doi:10.1152/jappl.1988.65.1.478.

Sawilowsky, S. 2009. New Effect Size Rules of Thumb. Theor. Behav. Found. Educ. Fac. Publ. Available from https://digitalcommons.wayne.edu/coe_tbf/4.

Söderström, T., Stefanovska, A., Veber, M., and Svensson, H. 2003. Involvement of sympathetic nerve activity in skin blood flow oscillations in humans. Am. J. Physiol. Heart Circ. Physiol. 284(5): H1638-1646. doi:10.1152/ajpheart.00826.2000.

Stauss, H.M., Anderson, E.A., Haynes, W.G., and Kregel, K.C. 1998. Frequency response characteristics of sympathetically mediated vasomotor waves in humans. Am. J. Physiol. 274(4 Pt 2): H1277-1283.

Stefanovska, A., Bracic, M., and Kvernmo, H.D. 1999. Wavelet analysis of oscillations in the peripheral blood circulation measured by laser Doppler technique. IEEE Trans. Biomed. Eng. 46(10): 12301239.

Tew, G.A., George, K.P., Cable, N.T., and Hodges, G.J. 2012. Endurance exercise training enhances cutaneous microvascular reactivity in post-menopausal women. Microvasc. Res. 83(2): 223-228. doi:10.1016/j.mvr.2011.09.002.

Wong, B.J., and Fieger, S.M. 2010. Transient receptor potential vanilloid type-1 (TRPV-1) channels contribute to cutaneous thermal hyperaemia in humans. J. Physiol. 588(Pt 21): 4317-4326. doi:10.1113/jphysiol.2010.195511.

Wong, B.J., Wilkins, B.W., Holowatz, L.A., and Minson, C.T. 2003. Nitric oxide synthase inhibition does not alter the reactive hyperemic response in the cutaneous circulation. J. Appl. Physiol. Bethesda Md 1985 95(2): 504-510. doi:10.1152/japplphysiol.00254.2003.

Zhao, J.L., Pergola, P.E., Roman, L.J., and Kellogg, D.L. 2004. Bioactive nitric oxide concentration does not increase during reactive hyperemia in human skin. J. Appl. Physiol. Bethesda Md 1985 96(2): 628632. doi:10.1152/japplphysiol.00639.2003. 


\section{Figure Legends}

Figure 1. Outline of the experimental protocol.

Figure 2. The mean responses in absolute CVC (A) and CVC normalized to max (B) during passive rest at thermoneutral conditions $\left(33^{\circ} \mathrm{C}\right)$ and during local skin heating to $39^{\circ} \mathrm{C}$ and $44^{\circ} \mathrm{C}$. ${ }^{*}$ indicates $p<0.05 . n=12$.

Figure 3. Mean skin blood flow and cardiovascular measures for the isometric handgrip exercise. Note that $n=12$ for all measure except MAP and cardiac output for children $(n=8)$ due to equipment failure. * indicates $p<0.05$ compared to adults; ${ }^{\$}$ indicates $p<0.05$ compared to baseline; \% indicates $p<0.05$ compared to exercise. 
Tables

Table 1. Participant characteristics ( $n=12$ per group)

\begin{tabular}{lll}
\hline & Boys & Men \\
\hline Age, $\mathrm{y}$ & $9 \pm 1$ & $21 \pm 1^{*}$ \\
Stature, m & $1.42 \pm 12$ & $1.76 \pm 0.10^{*}$ \\
Mass, $\mathrm{kg}$ & $35 \pm 9$ & $81 \pm 11^{*}$ \\
$\mathrm{BMI}, \mathrm{kg} \cdot \mathrm{m}^{2}$ & $17 \pm 2$ & $25 \pm 2^{*}$ \\
$\dot{V} \mathrm{O} 2 \mathrm{max}, \mathrm{ml} \cdot \mathrm{kg}^{-1} \cdot \mathrm{min}^{-1}$ & $51 \pm 5$ & $46 \pm 9$ \\
Resting measures & & \\
$\quad$ Systolic blood pressure, mm Hg & $89 \pm 4$ & $112 \pm 5^{*}$ \\
$\quad$ Diastolic blood pressure, mm Hg & $52 \pm 4$ & $70 \pm 3^{*}$ \\
Heart rate, bpm & $64 \pm 5$ & $66 \pm 5$ \\
$\dot{Q}, \mathrm{~L} \cdot \mathrm{min}^{-1}$ & $2.98 \pm .0 .28$ & $4.38 \pm 0.26^{*}$ \\
\hline
\end{tabular}

* indicates $p<0.05$ compared to boys. Note that cardiac output was only obtained in $n=8$ children.

Table 2. Local heating laser-Doppler flux and wavelet data ( $n=12$ per group)

\begin{tabular}{|c|c|c|c|c|c|c|}
\hline & \multicolumn{2}{|c|}{$33^{\circ} \mathrm{C}$} & \multicolumn{2}{|c|}{$39^{\circ} \mathrm{C}$} & \multicolumn{2}{|c|}{$44^{\circ} \mathrm{C}$} \\
\hline & Boys & Men & Boys & Men & Boys & Men \\
\hline LDF, PU & $16.57 \pm 1.06$ & $8.41 \pm 0.91 *$ & $32.94 \pm 4.39$ & $22.3 \pm 2.9^{*}$ & $150.9 \pm 14.5$ & $162.5 \pm 17.1$ \\
\hline Endo NO-Indep, \% & $20.2 \pm 1.3$ & $15.1 \pm 1.6^{*}$ & $25.8 \pm 2.1^{\dagger}$ & $21.4 \pm 2.5^{* \dagger}$ & $8.0 \pm 0.6^{\dagger}$ & $7.5 \pm 0.5^{\dagger}$ \\
\hline Endo NO-Dep, \% & $20.9 \pm 1.2$ & $12.1 \pm 1.6^{*}$ & $30.4 \pm 1.2^{\dagger}$ & $26.5 \pm 1.5^{* \dagger}$ & $8.3 \pm 0.4^{\dagger}$ & $9.3 \pm 0.9^{\dagger}$ \\
\hline Neurogenic, $\%$ & $13.5 \pm 0.7$ & $19.6 \pm 1.7^{*}$ & $12.1 \pm 1.1$ & $12.6 \pm 1.0^{\dagger}$ & $15.8 \pm 0.9^{\dagger}$ & $14.2 \pm 1.4^{\dagger}$ \\
\hline Myogenic, \% & $10.2 \pm 2.5$ & $18.3 \pm 1.4^{*}$ & $10.6 \pm 0.8$ & $12.7 \pm 1.3^{\dagger}$ & $19.6 \pm 1.3^{\dagger}$ & $20.8 \pm 1.1^{\dagger}$ \\
\hline Respiratory, \% & $11.8 \pm 1.6$ & $17.6 \pm 1.4^{*}$ & $10.8 \pm 1.7$ & $13.9 \pm 1.9^{\dagger}$ & $18.3 \pm 0.9^{\dagger}$ & $17.3 \pm 1.0^{\dagger}$ \\
\hline Cardiac, \% & $23.4 \pm 1.9$ & $17.2 \pm 1.3^{*}$ & $10.3 \pm 1.3^{+}$ & $12.9 \pm 1.6^{\dagger}$ & $30.1 \pm 1.1^{\dagger}$ & $30.9 \pm 1.4^{\dagger}$ \\
\hline
\end{tabular}

* indicates $p<0.05$ compared to boys; $\dagger$ indicates $p<0.05$ compared to $33^{\circ} \mathrm{C}$. Data are mean $\pm 95 \% \mathrm{CI}$.

Table 3. Maximal, time-to-peak, and area-under-the-curve post occlusive reactive hyperaemic responses in boys and men ( $n=12$ per group)

\begin{tabular}{lll}
\hline & Boys & Men \\
\hline Max CVC (PU.mm Hg-1) & $0.549 \pm 0.035$ & $0.546 \pm 0.054$ \\
Max CVC (\%max) & $27.9 \pm 1.4$ & $28.9 \pm 2.0$ \\
Time-to-peak (s) & $33.3 \pm 4.7$ & $10.7 \pm 1.9^{*}$ \\
AUC, CVC. s $^{-1}$ & $4035 \pm 456$ & $2336 \pm 438^{*}$ \\
\hline
\end{tabular}

* indicates $p<0.05$ compared to boys

Table 4. Laser-Doppler flux and wavelet analysis for max post occlusive reactive hyperaemia ( $n=12$ per group)

\begin{tabular}{lll}
\hline & Boys & Men \\
\hline LDF, PU & $41.7 \pm 2.65$ & $45.2 \pm 3.8$ \\
Endo NO-Indep, \% & $23.7 \pm 1.0$ & $20.8 \pm 1.5^{*}$ \\
Endo NO-Dep, \% & $28.3 \pm 0.9$ & $23.8 \pm 0.8^{*}$ \\
Neurogenic, \% & $16.9 \pm 0.8$ & $23.1 \pm 0.7^{*}$ \\
Myogenic, \% & $15.2 \pm 1.1$ & $14.7 \pm 0.8$ \\
Respiratory, \% & $6.4 \pm 0.3$ & $10.0 \pm 0.9^{*}$ \\
Cardiac, \% & $9.5 \pm 0.8$ & $7.5 \pm 0.5^{*}$ \\
\hline
\end{tabular}

* indicates $p<0.05$ compared to boys 
Table 5. Wavelet data in response to isometric handgrip exercise $(n=12)$

\begin{tabular}{lllll}
\hline & Boys & \multicolumn{3}{l}{ Men } \\
\hline & Baseline & Exercise & Baseline & Exercise \\
Endo NO-Indep, \% & $12.1 \pm 1.1$ & $22.3 \pm 2.7 \dagger$ & $17.2 \pm 1.6^{*}$ & $28.4 \pm 3.8^{*} \dagger$ \\
Endo NO-Dep, \% & $10.7 \pm 1.1$ & $13.2 \pm 1.9$ & $17.6 \pm 1.7^{*}$ & $17.9 \pm 2.4^{*}$ \\
Neurogenic, \% & $13.6 \pm 0.9$ & $12.4 \pm 0.9$ & $19.6 \pm 1.7^{*}$ & $19.8 \pm 2.3^{*}$ \\
Myogenic, \% & $21.3 \pm 3.1$ & $17.4 \pm 2.4$ & $17.8 \pm 1.3$ & $14.1 \pm 2.1^{*}$ \\
Respiratory, \% & $18.9 \pm 1.5$ & $16.7 \pm 2.5$ & $12.2 \pm 1.4^{*}$ & $8.3 \pm 1.0^{*} \dagger$ \\
Cardiac, \% & $23.7 \pm 1.8$ & $17.8 \pm 1.4 \dagger$ & $15.1 \pm 1.3^{*}$ & $11.4 \pm 2.0^{*}$ \\
\hline
\end{tabular}

$*$ indicates $p<0.05$ compared to boys, $\dagger$ indicates $p<0.05$ compared to baseline 


\section{Figure 1. Outline of protocol}

\begin{tabular}{|c|c|c|c|c|c|c|c|c|c|}
\hline Phase & Baseline & IHE & Recovery & PORH & Recovery & $\Delta T_{\text {loc }}$ & Stabilize & $\Delta T_{\text {loc }}$ & Stabilize \\
\hline Duration (min) & 20 & 2 & 5 & 3 & 15 & 2 & 25 & 5 & 25 \\
\hline $\mathbf{T}_{\text {loc }}$ & \multicolumn{5}{|c|}{$33^{\circ} \mathrm{C}$} & \multicolumn{2}{|r|}{$39^{\circ} \mathrm{C}$} & \multicolumn{2}{|c|}{$44^{\circ} \mathrm{C}$} \\
\hline
\end{tabular}

IHE = isometric handgrip exercise

PORH = post occlusive reactive hyperaemia

$\mathrm{T}_{\text {loc }}=$ local skin temperature 

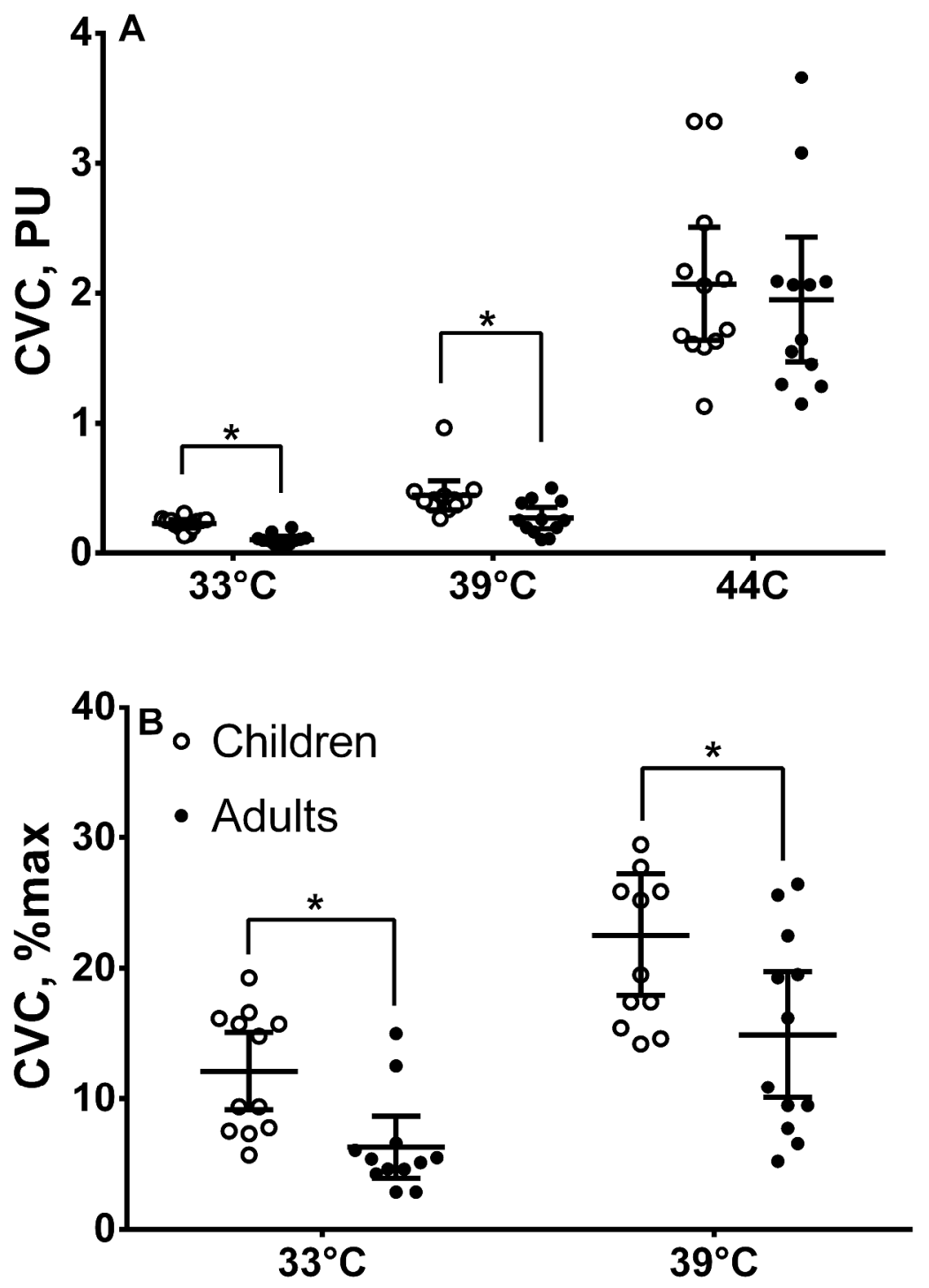

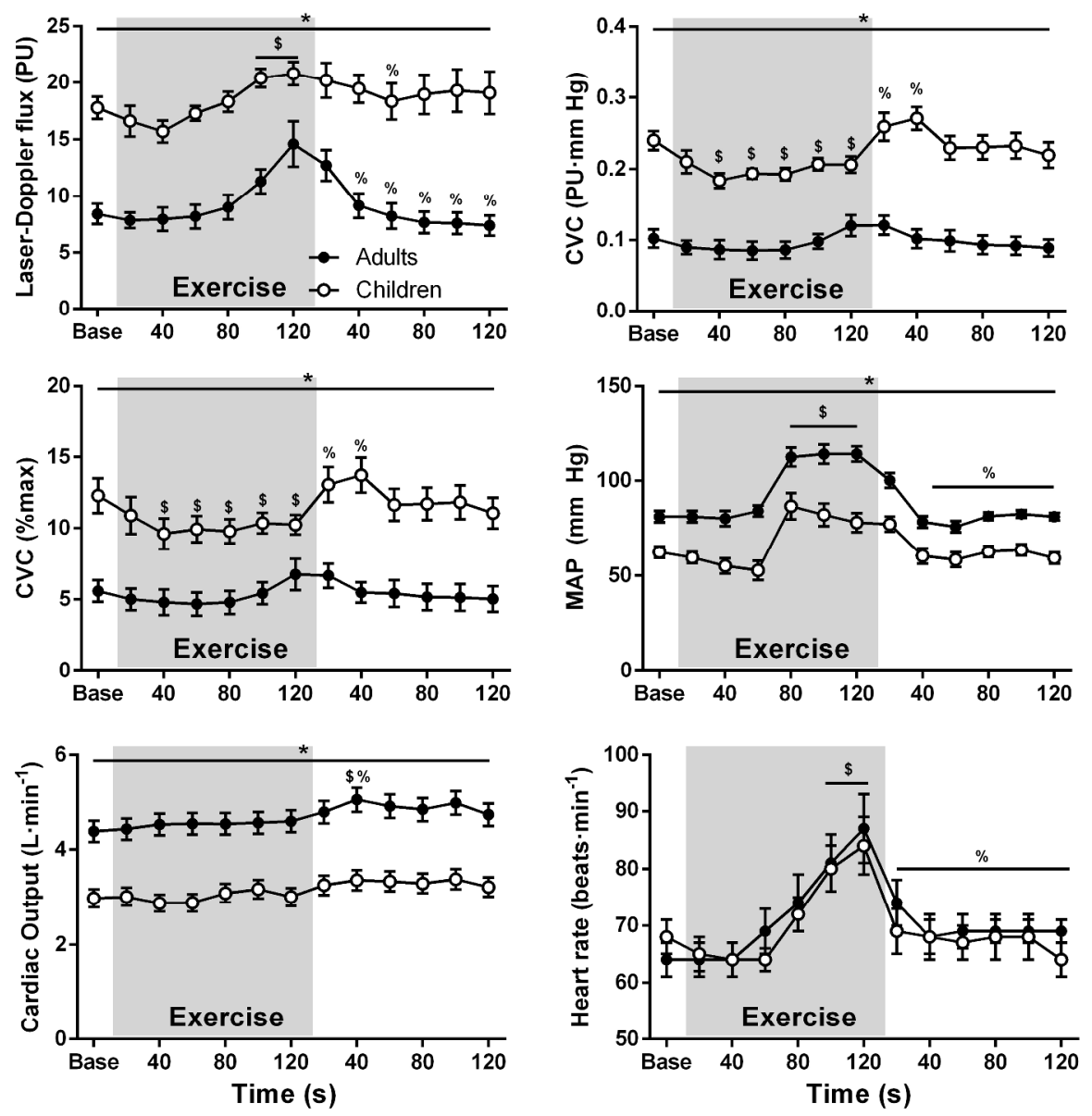